\title{
Evaluación de la velocidad de corrosión del acero al carbono mediante técnicas electroquímicas
}

\author{
Corrosion rate evaluation of the carbon steel \\ trough electrochemical techniques
}

\author{
Jeimmy González-Masis's \\ Luis Garita-Arce ${ }^{2}$
}

2 Licenciado en Ingeniería Química de la Universidad de Costa Rica. Teléfono: (506) 2297-4020. Correo electrónico: Igaritaar@ice.go.cr. Investigador en el Centro de Investigación en Corrosión (ClCorr) del Instituto Costarricense de Electricidad (ICE). Costa Rica. 


\section{Palabras clave}

Ruido electroquímico (RE); resistencia a la polarización lineal (RPL); monitor electroquímico de corrosión atmosférica (MECA); humedad relativa crítica.

\section{Resumen}

Usualmente los estudios de corrosión atmosférica se caracterizan por su larga duración, meses e incluso años. Sin embargo, se han desarrollado las técnicas electroquímicas, recientes en comparación con otros métodos y que permiten obtener datos en tiempo real, incluyendo velocidad de corrosión. En esta investigación se valoran los ensayos de ruido electroquímico y resistencia a la polarización lineal, de manera que se analizaron los datos obtenidos, se establecieron relaciones entre la forma de los gráficos y el tipo de corrosión, así como la relación entre los datos de corrosión y las condiciones atmosféricas para hallar, finalmente, que existe un comportamiento más acorde cuando se utiliza el ensayo de resistencia a la polarización lineal con el monitor electroquímico tres electrodos tipo peineta.

\section{Key words}

Electrochemical noise (EN); lineal polarization resistance (LPR); electrochemical monitor of atmospheric corrosion; critical relative humidity.

\begin{abstract}
Usually the atmospheric corrosion studies are characterized by their long duration, months and even years. However electrochemical techniques have been developed, recent in comparison to other methods, allowing obtain real-time data, including corrosion rate. In this research electrochemical noise and lineal polarization resistance tests are valued, so obtained data were analyzed, relations were established between the graphics form and the corrosion type, as well as the relationship between the corrosion data and atmospheric conditions, to find, finally, there is a more consistent behavior when the lineal polarization resistance test is used with the three comb-type electrodes electrochemical monitor.
\end{abstract}

\section{Introducción}

El mecanismo de corrosión atmosférica es de naturaleza electroquímica, donde tienen lugar dos reacciones que ocurren en lugares distintos: ánodos y cátodos locales. El circuito eléctrico se complementa por el movimiento de aniones y cationes en el electrolito (medio líquido conductor).

El espesor de ese electrolito o capa de humedad juega un papel muy importante, en tanto que para espesores menores que 10 $\mu \mathrm{m}$ las velocidades de corrosión son prácticamente despreciables, conforme crece el espesor de la película, aumenta la velocidad de corrosión hasta un punto máximo, aproximadamente $100 \mu \mathrm{m}$. Para espesores mayores la velocidad de corrosión más bien disminuye porque el acceso del oxígeno desde la atmósfera hasta la superficie se dificulta (Otero, 200 I).

En esta investigación se pretende evaluar la velocidad de corrosión del acero al carbono ASTM A36, aplicando las técnicas ruido electroquímico (RE) y resistencia a la polarización lineal (RPL), mediante diferentes monitores electroquímicos expuestos en condiciones de humedad relativa y temperatura del laboratorio.

El ensayo de ruido electroquímico estudia las variaciones espontáneas en corriente y voltaje (conocidas como ruido) producidas por micropilas que se forman sobre la superficie del metal, y que preceden a la reacción electroquímica durante el proceso de corrosión (Arriaga, R., Malo, J., Tres, G., y Uruchurtu, J., 200 I), por tanto no se requiere perturbar el sistema. El ensayo de resistencia a la polarización lineal, por su parte, consiste en aplicar un sobrepotencial respecto al potencial de equilibrio, para percibir la respuesta del electrodo frente a las reacciones de oxidación y de reducción en estas condiciones, y así conocer la evolución de la corrosión en el tiempo, haciendo uso de la ecuación Stern-Geary:

$$
\text { icorr }=\frac{b a \cdot b c}{R p \cdot 2.3(b a+b c)}
$$


donde:

icorr: densidad de corriente de corrosión

Rp: resistencia a la polarización

ba, bc: constantes de Tafel, (constantes

empíricas para el metal)

Para realizar estas mediciones, se requieren unos dispositivos denominadas monitores electroquímicos de corrosión atmosférica (MECA), que pueden tener diferentes configuraciones, entre ellas se encuentran las probetas de tres electrodos idénticos y tres electrodos tipo peineta. Ambas poseen tres láminas de acero separadas entre sí por un aislante, una de las láminas (central) no se polariza y funciona como electrodo de referencia, las otras dos funcionan como electrodos de trabajo. La diferencia fundamental entre estos monitores es la forma y disposición de las láminas con que son construidos (figura I).

Además estos MECA requieren de una capa de humedad en su superficie, que funcione como electrolito. Esto es indispensable principalmente cuando la humedad relativa atmosférica es muy baja, ya que para ciertos valores (según sea el metal) la velocidad de corrosión puede resultar casi despreciable (Otero, 200 I). A estos valores de humedad se les conoce como humedad relativa crítica. En el caso del acero por ejemplo, se puede considerar un valor de 60\% (Genescá, Salvador, Mariaca, y Uruchurtu, 1999). Por eso es necesario utilizar sustancias activadoras, que mantengan una capa de electrolito sobre la superficie y aseguren el cierre del circuito que se forma bajo cualquier condición de humedad; entre las más comunes están el hidróxido de sodio y el hidróxido de potasio, sustancias higroscópicas y corrosivas.

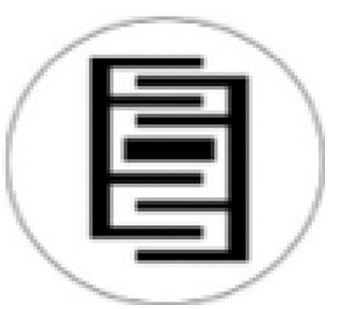

(a)

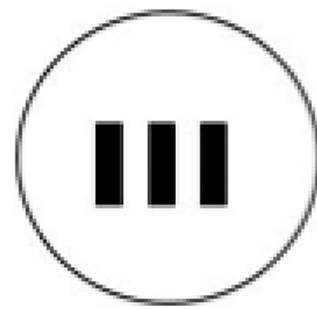

(b)
Figura I. (a) Configuración del MECA tres electrodos tipo idénticos. (b) Configuración del MECA tres electrodos idénticos.

\section{Materiales y métodos}

Para emplear las técnicas electroquímicas RPL y RE, se construyeron dos monitores electroquímicos de configuraciones distintas: uno de tres electrodos tipo peineta y otro de tres electrodos idénticos. Se montaron tres pequeñas láminas de acero al carbono ASTM A36 separadas entre sí por un material aislante, y embebidas en una resina poliéster, en donde una cara de los electrodos se dejó expuesta al ambiente. La mezcla de la resina y las placas se depositaron en un molde y se dejaron secar durante seis horas. De esta manera, el monitor de tres electrodos idénticos resultó con un área de aproximadamente $0,33 \mathrm{~cm}^{2}$, y el área del monitor de tres electrodos tipo peineta fue $3,69 \mathrm{~cm}^{2}$.

Una vez listos, los monitores se lijaron y se limpiaron; posteriormente se activaron en dos ocasiones, dejándolos reposar inmersos en una solución alcalina de 0,I M de hidróxido de sodio, a una temperatura de $80^{\circ} \mathrm{C}$ sin agitación para la primera activación, y entre $85^{\circ} \mathrm{C}$ y $90^{\circ} \mathrm{C}$ con agitación para la segunda activación, y se dejaron secar por un lapso de aproximadamente dos horas, previo a la realización de los ensayos.

A partir de ahí se ejecutaron las pruebas de manera continua, realizando un análisis mediante inspección directa de los registros experimentales, para detener las pruebas en el momento en que se observe un comportamiento atípico. Se utilizó un potenciostato galvanostato de la marca ACM Instruments, Field Machine y se registraron los datos de temperatura y humedad relativa con un termohigrómetro.

Para la técnica de resistencia de polarización lineal se empleó un barrido de potencial desde los $-20 \mathrm{mV}$ hasta $20 \mathrm{mV}$, con respecto al potencial de circuito abierto, con una velocidad de barrido de $10 \mathrm{mV}$ por minuto, mientras que para el ensayo de ruido electroquímico se registraron los datos cada segundo, con un total de 1024 lecturas en cada prueba.

\section{Resultados y discusión}

Esta sección se divide en dos partes, tomando en cuenta que se realizaron dos activaciones hidrofilicas en total: primera corrida y segunda corrida de datos.

\section{Primera corrida de datos}

Los primeros ensayos de ruido electroquímico muestran comportamientos distintos entre las pro- 
betas, al analizar el trazo de la figura 2 se puede apreciar la pequeña amplitud de las fluctuaciones, lo cual se relaciona con el comportamiento de los sistemas pasivos, según el análisis propuesto por Aballe et al. (2002); el monitor tres electrodos idénticos, por el contrario, muestra tránsitos con mayores variaciones (figura 3), por tanto se puede asociar estos gráficos con fenómenos de corrosión localizada.

En cuanto a los primeros ensayos de resistencia a la polarización lineal, los gráficos también son muy distintos. Para el MECA tres electrodos tipo peineta, la corriente aumenta casi proporcionalmente con el potencial (figura 4), lo que se aproxima a una curva teórica para el ensayo de RPL, pues tal como indican Corte y Peña (20 I0), la línea recta es ideal. Por el contrario, para el MECA tres electrodos idénticos la corriente y el potencial aplicado no varían conjuntamente, esto podría deberse a que el sobrepotencial es mucho mayor al potencial de equilibrio, tal como menciona Corte y Peña (20l0).

La última prueba realizada con el MECA tres electrodos tipo peineta fue RPL (figura 5), luego de aproximadamente 58 horas de haber realizado el primer ensayo. A partir de esta no se realizaron más corridas, debido al comportamiento atípico de la
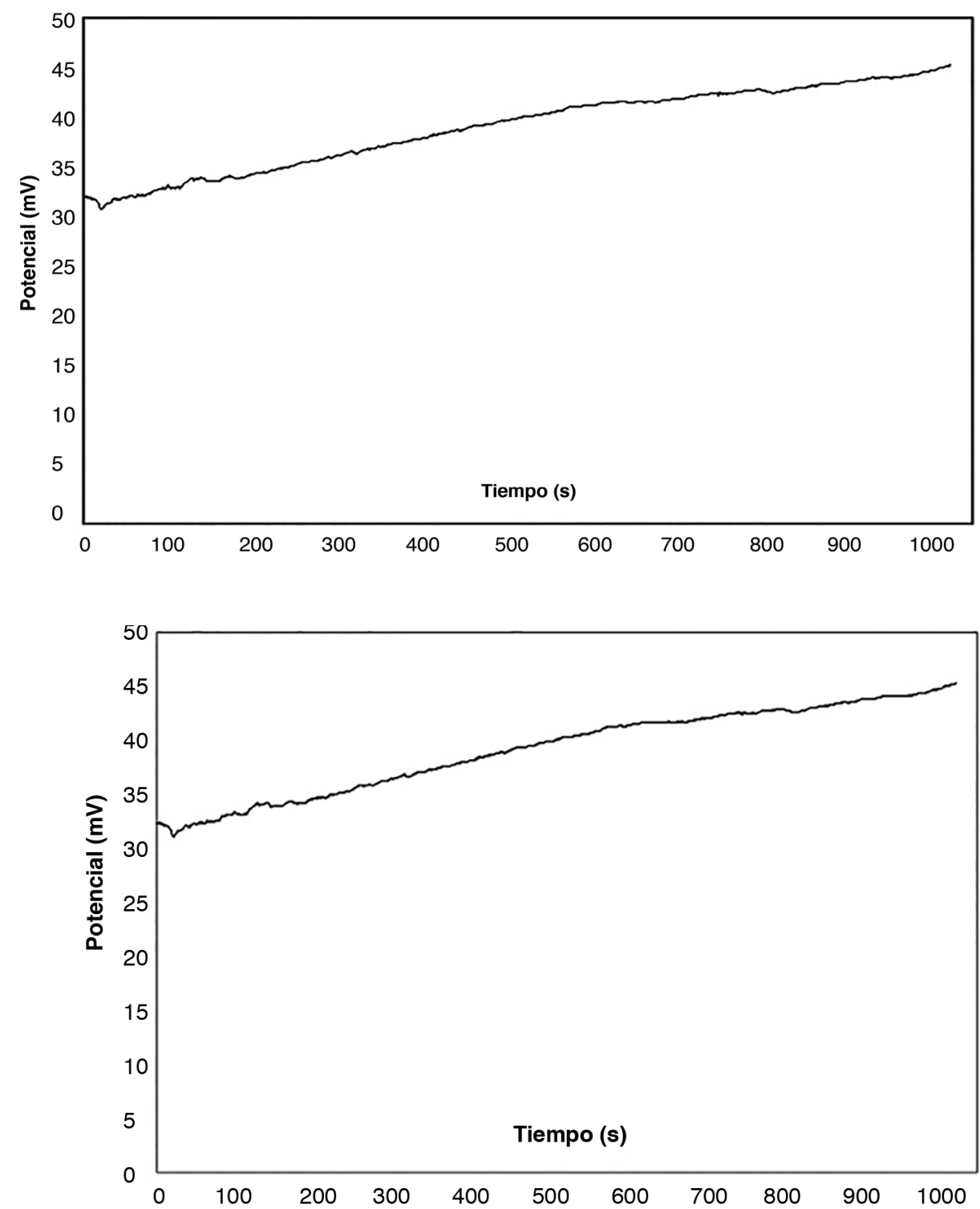

Figura 2. Registro de corriente y voltaje en el tiempo, ensayo RE para el MECA tres electrodos tipo peineta. 

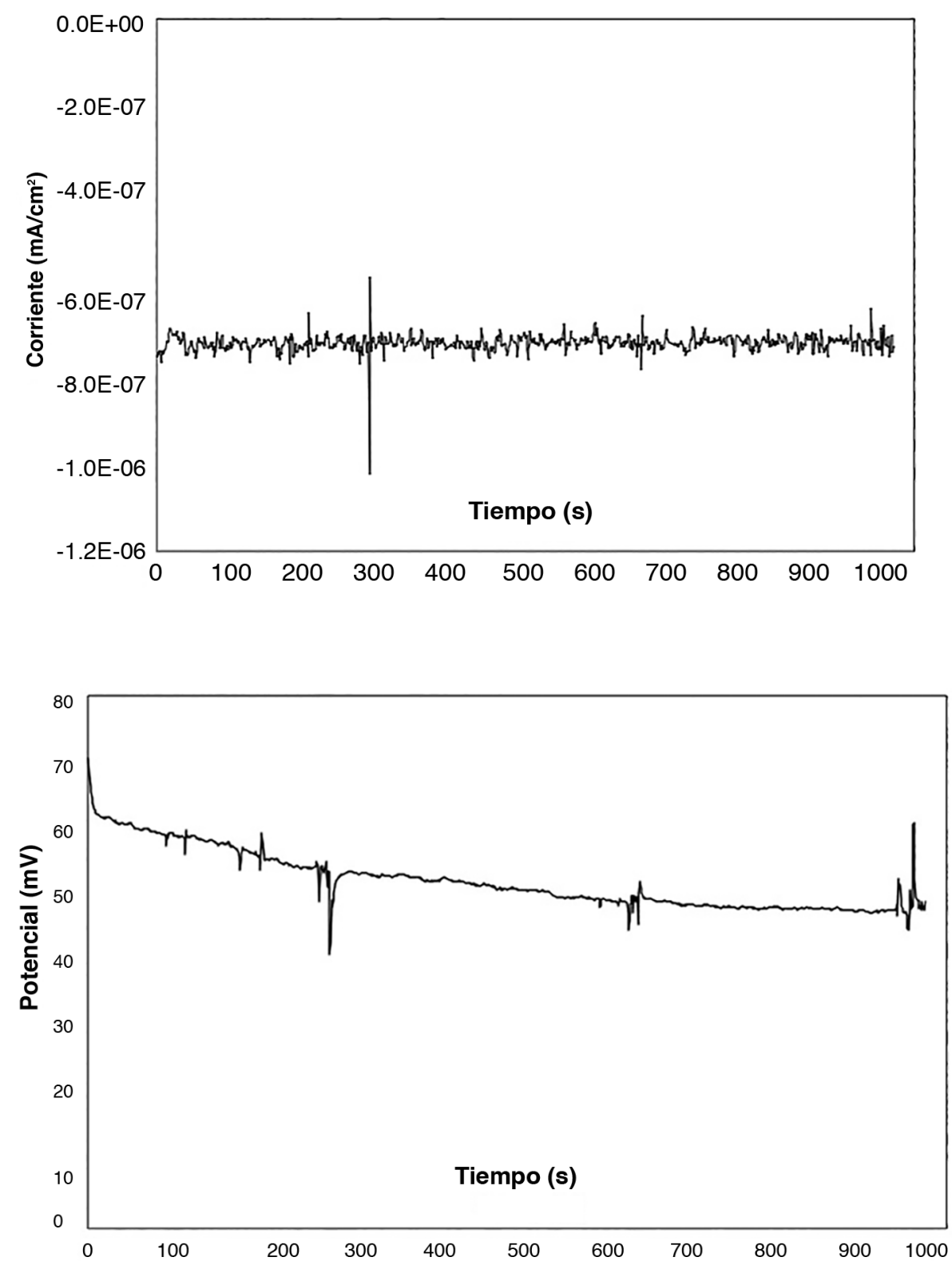

Figura 3. Registro de corriente y voltaje en el tiempo, ensayo RE para el MECA tres electrodos idénticos.

curva. Se lograron realizar 22 ensayos en total con este monitor, más eficiente si se compara con el monitor tres electrodos idénticos, con el que sólo se lograron realizar 17 ensayos durante 50 horas aproximadamente.

En cuanto a las condiciones atmosféricas, es importante resaltar, a partir de las figuras 6 y 7 , que el valor de resistencia de polarización (Rp) y de resistencia al ruido $(R n)$, tienden a variar con los cambios de humedad relativa, de manera que cuando ésta dis- minuye, las resistencias aumentan y simultáneamente disminuye la velocidad de corrosión.

De esta manera, en el gráfico de la figura 6 se observa, aproximadamente a las 18 horas, el mayor valor de humedad relativa registrada en la serie de datos (66\%) y el valor de resistencia a la polarización lineal más bajo; a ese mismo punto corresponde, en el gráfico de la figura 8, la mayor velocidad de corrosión registrada. En esa misma figura también se puede apreciar una disminución en la velocidad de corrosión, según la línea de tendencia. 


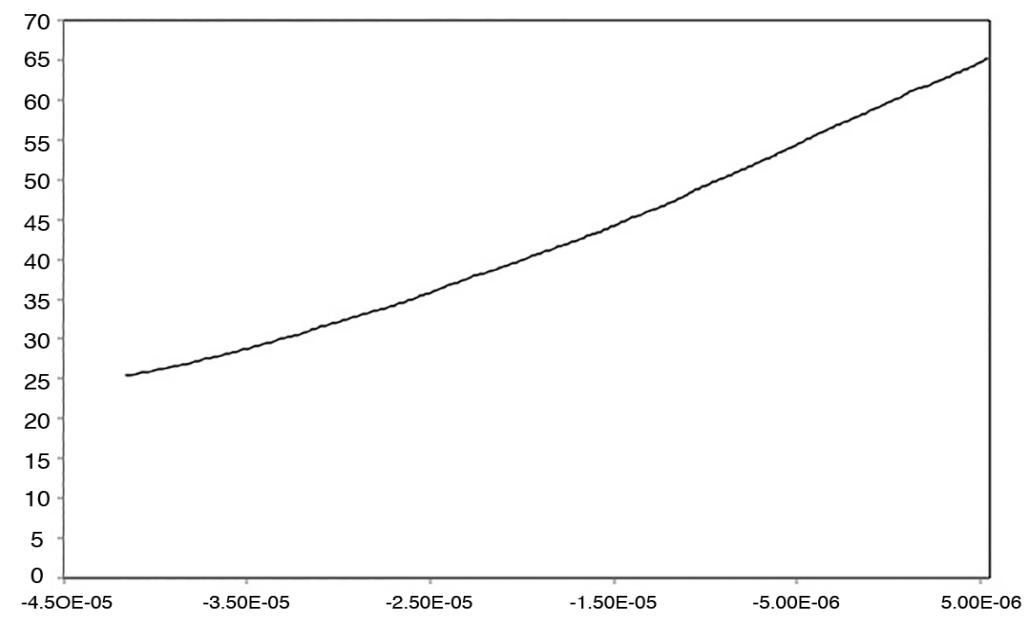

Figura 4. Curva generada en el ensayo RPL para el MECA tres electrodos tipo peineta.

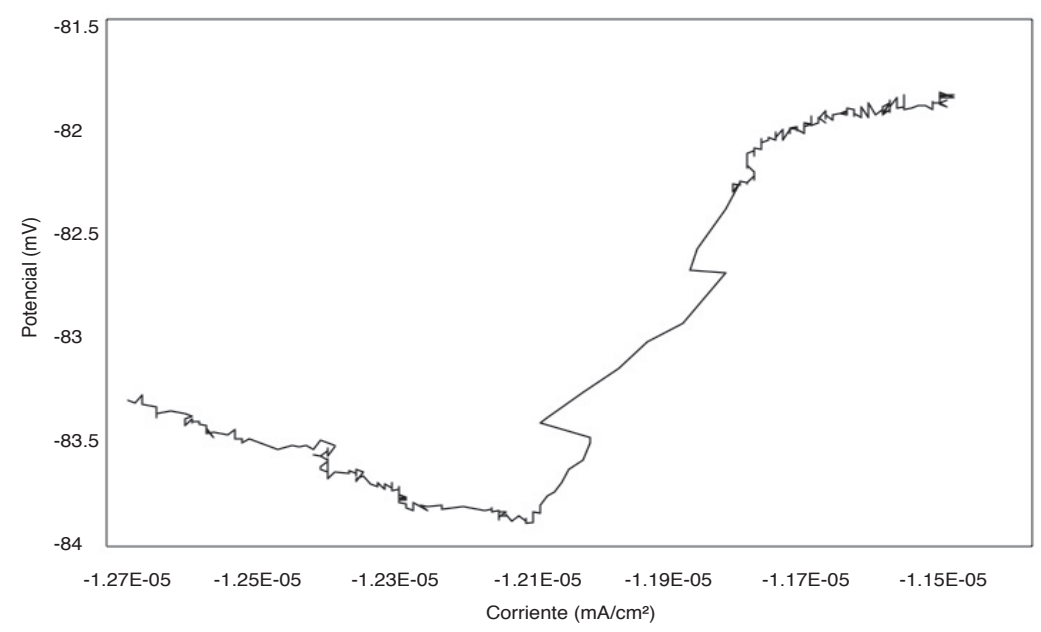

Figura 5. Curva generada en el ensayo RPL para el MECA tres electrodos tipo peineta.

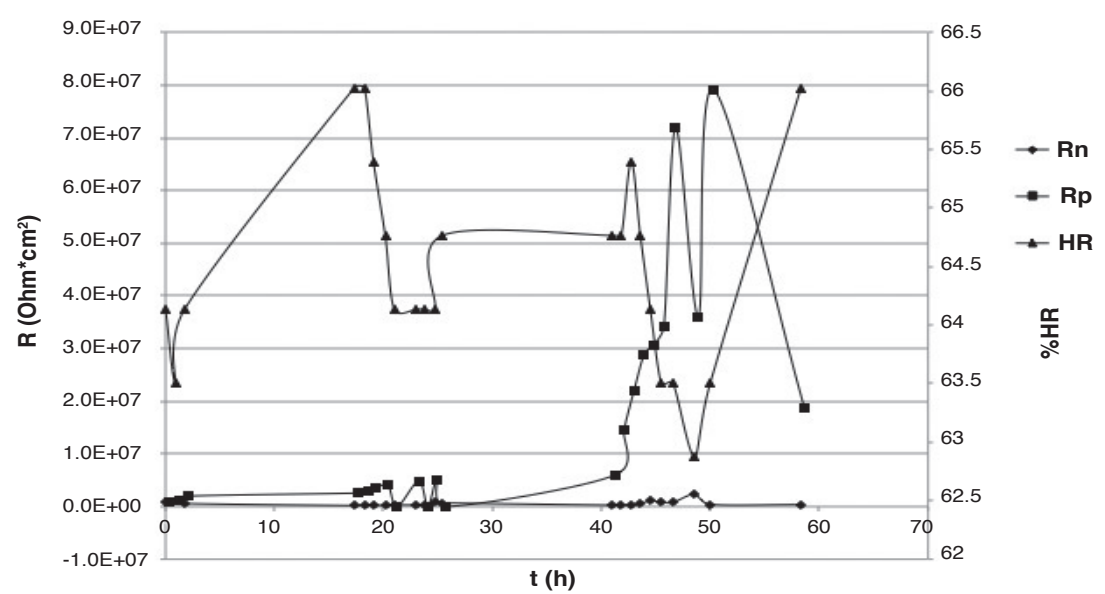

Figura 6. Resistencia de polarización, resistencia al ruido y humedad relativa con respecto al tiempo para el MECA tres electrodos tipo peineta. 


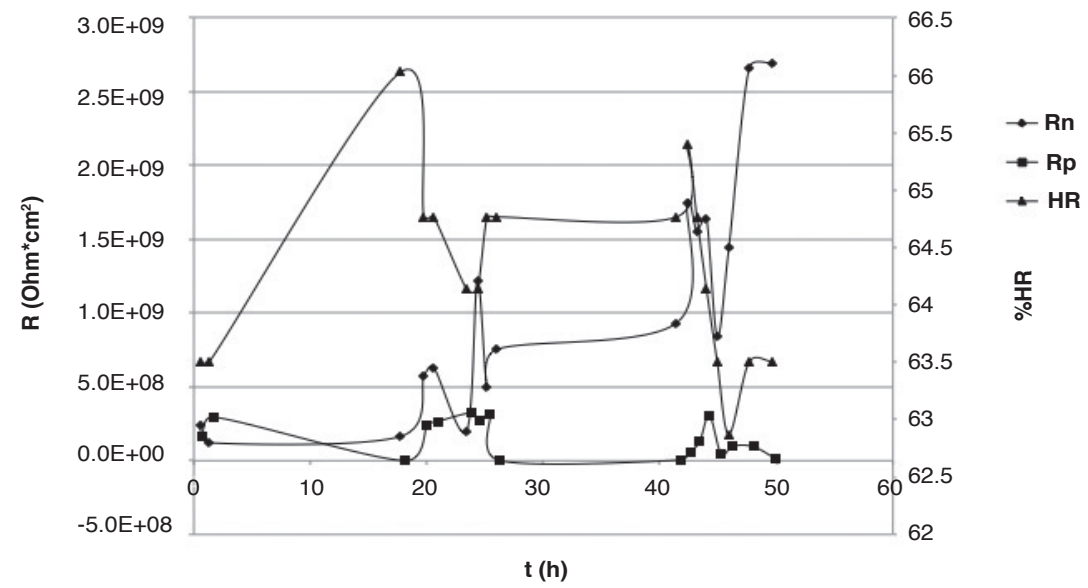

Figura 7. Resistencia de polarización, resistencia al ruido y humedad relativa con respecto al tiempo para el MECA tres electrodos idénticos.

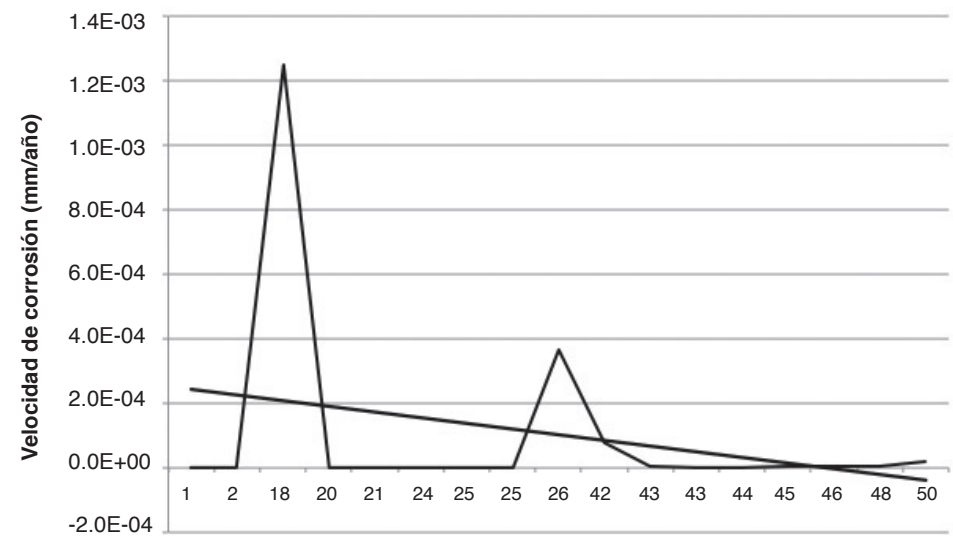

t (h)

Figura 8. Velocidad de corrosión en el tiempo para el MECA tres electrodos idénticos, ensayo de RPL.

\section{Segunda corrida de datos}

Los ensayos iniciales de RE demuestran que en ambas probetas podrían tener lugar fenómenos de corrosión localizada, debido a que las variaciones son pequeñas, pero perceptibles.

En cuanto a los ensayos de RPL es importante hacer notar que para las pruebas iniciales luego de la segunda activación, se obtuvieron resultados que se encuentran dentro de los parámetros típicos, a diferencia de los eventos mostrados en los gráficos de los últimos ensayos de la primera corrida de datos, tal como se observa al comparar las figuras 5 (correspondiente a la primera activación) y 9, lo cual hace suponer que ya la probeta había perdido el carácter hidrofilico hacia el final de esa primera corrida.

La probeta de tres electrodos idénticos fue la primera en presentar alteraciones evidentes en los curvas de los distintos gráficos, sobretodo de los ensayos de RPL, por eso se realizaron tan sólo 32 ensayos con este monitor, mientras que con el MECA tres electrodos tipo peineta se realizaron 100 ensayos.

En la figura 10, para la última prueba de RPL, el comportamiento atípico del MECA nuevamente se puede relacionar con la pérdida de su carácter hidrofilico.

Parece ser que la segunda activación de las probetas fue más exitosa que la primera porque con la primera activación sólo se pudieron realizar ensayos 


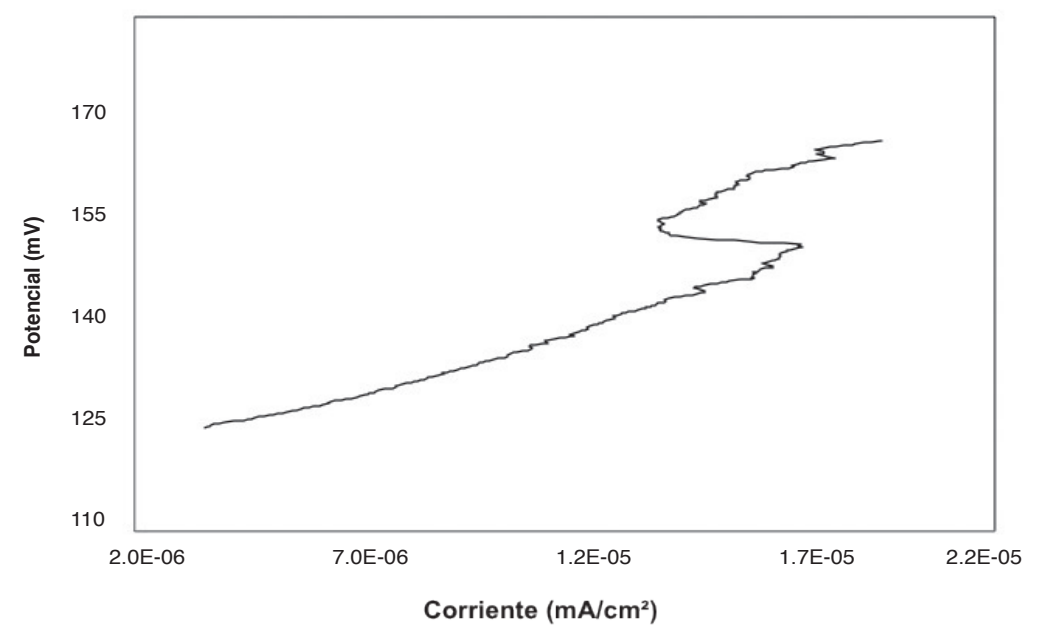

Figura 9. Curva generada en el ensayo RPL para el MECA tres electrodos tipo peineta.

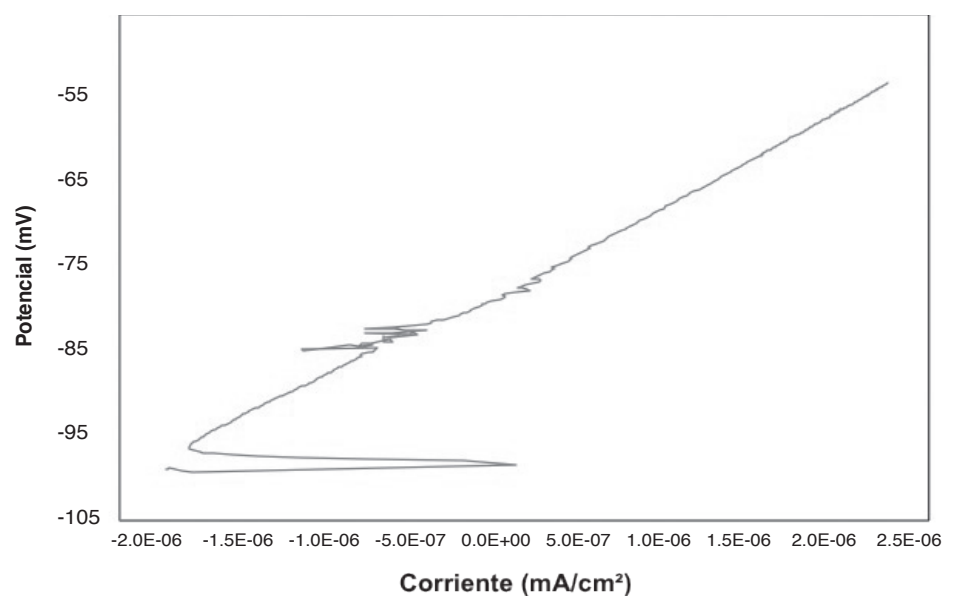

Figura 10. Curva generada en el ensayo de RPL para el MECA tres electrodos tipo peineta.

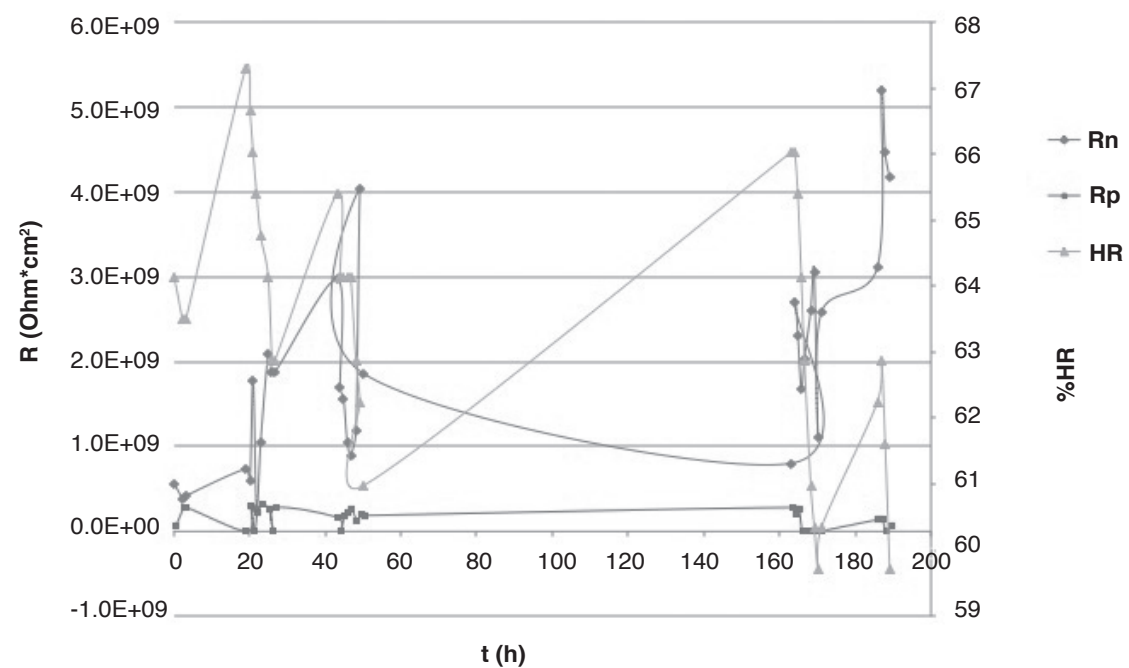

Figura II. Resistencia a la polarización, resistencia al ruido y humedad relativa con respecto al tiempo para el MECA tres electrodos idénticos. 


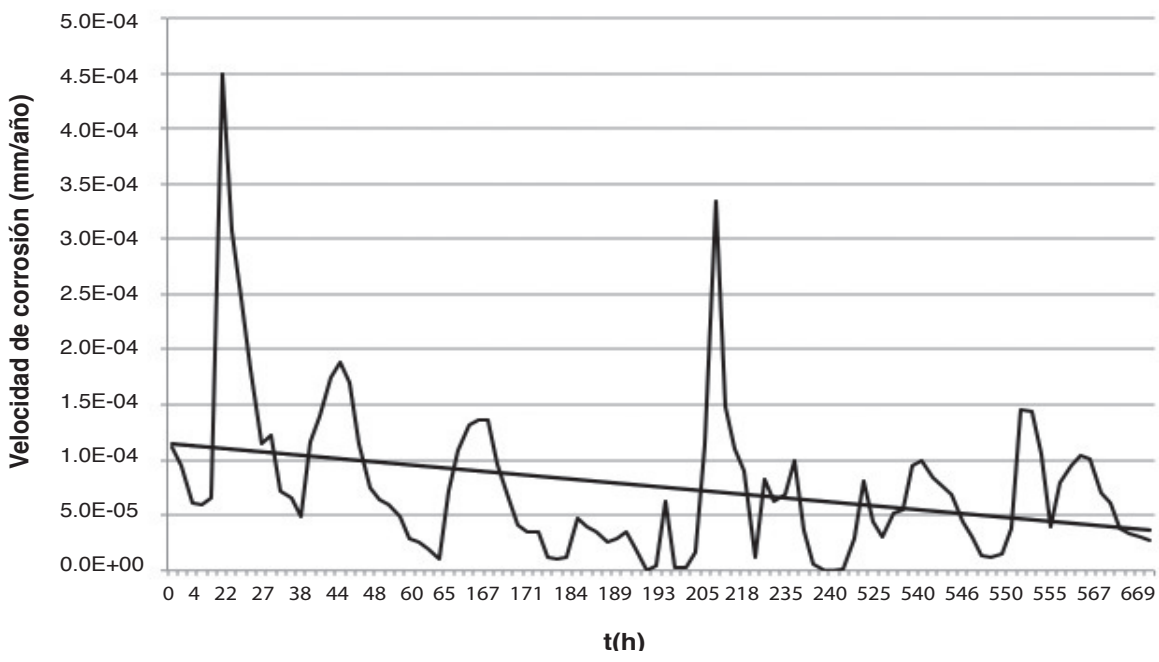

Figura 12. Velocidad de corrosión en el tiempo para el MECA tres electrodos peineta, ensayo de RPL.

durante un máximo de 58 horas aproximadamente, mientras que para la segunda activación se realizó pruebas durante casi 672 horas. Entre los motivos que pudieron haber influido para que esto sucediera, se encuentran las condiciones de activación, ya que la temperatura de activación se aumentó de 5 a $10{ }^{\circ} \mathrm{C}$ para la segunda ocasión, y se efectuó una agitación continua de la sustancia.

Al analizar las condiciones atmosféricas, un aspecto importante a resaltar es que, tal como en la primera activación, los gráficos reflejan la tendencia de mayor afinidad entre la probeta de tres electrodos tipo peineta y el ensayo de RPL, pues los datos de resistencia a la polarización (Rp) son los que sufren mayores variaciones durante esta prueba, de manera análoga, para el MECA de tres electrodos idénticos, la resistencia al ruido $(\mathrm{Rn})$ es la que sufre mayores variaciones en relación con Rp. Esta tendencia se puede observar en el gráfico de la figura II (y figuras 6 y 7 para la primera corrida).

Además, nuevamente se demuestra que conforme la humedad relativa aumenta, la resistencia (sea Rp o $\mathrm{Rn}$ ) disminuye, y por ende la velocidad de corrosión aumenta.

Al trazar líneas de tendencia central en los gráficos de distintas pruebas para ambos MECA, se observa que existe una ligera disminución en la tasa de corrosión, tal como se aprecia en la figura 12 .

Es probable que la disminución en la velocidad de corrosión que se observa en la figura 12, se deba a los productos de corrosión formados en la superficie de los MECA, los cuales se pueden observar a simple vista, dado que se acumulan y más bien llegan a comportarse como una especie de capa protectora, desacelerando el ataque (González, 1989).

\section{Conclusiones}

Con el MECA tres electrodos tipo peineta se obtienen los mejores resultados según las pruebas realizadas, especialmente si se aplican los ensayos de resistencia a la polarización lineal.

Si se emplea la técnica de ruido electroquímico, el MECA tres electrodos idénticos brinda mejores resultados que el MECA tres electrodos tipo peineta.

Durante el proceso de activación de las probetas, una temperatura de $85^{\circ} \mathrm{C}$ a $90^{\circ} \mathrm{C}$ y agitación continua provoca que la activación perdure por más tiempo (hasta 67| horas), con respecto a las condiciones de la primera corrida de datos, de $80^{\circ} \mathrm{C}$ sin agitación.

Ambos monitores electroquímicos responden a los cambios de las condiciones atmosféricas para ambos ensayos: en los puntos más bajos de humedad relativa, la resistencia es mayor (sea Rn o Rp), y la velocidad de corrosión disminuye. 


\section{Bibliografía}

Arriaga, R., Malo, J., Tres, G., y Uruchurtu, J. (200 I). Monitoreo por ruido electroquímico de la corrosión del acero en una atmósfera contaminada. Revista Internacional de Contaminación Ambiental, 17(4), 172-173.Obtenido desde: http://www.revistas.unam.mx/index.php/rica/article/ view/25358.

Aballe, A., Bárcena, M., Botana, J. (2002). Ruido Electroquímico. Métodos de análisis. Oviedo, España: Septem Ediciones

Bethencourt, M., Botana, F.J., González, L., y Sánchez, J.M. (2009). Medida de ruido electroquímico para el estudio de procesos de corrosión de aleaciones metálicas. Revista de Metalurgia, 45(2), |45-147. Obtenido desde: http://revistademetalurgia.revistas.csic.es/index.php/revistademetalurgia/ artiart/view/I58/I56
Corte, D., y Peña, J. (2010). Obtención de curvas galvanostáticas y ensayos de resistencia a la polarización en varillas de construcción ASTM A42 en solución de agua al 3,5\% de cloruro de sodio (Tesis de licenciatura). Obtenido desde: http://www.dspace.espol.edu.ec/handle//23456789// 0025

Genescá, J., Salvador, L., Mariaca, L., y Uruchurtu, J. (1999). Corrosividad atmosférica (MICAT-México). México: Plaza y Valdés.

González, J. (1989). Control de la corrosión: estudio y medida por técnicas electroquímicas. Madrid, España: GRAFIPREN, S.A.

Norma ASTM G 199-09. Standard Guide for Electrochemical Noise Measurement. Estados Unidos: ASTM International.

Norma ISO 9223-1992. Corrosion of metals and alloys. Corrosivity of atmospheres-Classification. Suiza: ISO.

Otero, E. (200I). Corrosión ydegradación de materiales (I Reimpresión). Madrid, España: Síntesis S.A. 